\title{
Tejiendo vínculos por la infancia
}

\author{
Valera, Luis Miguel \\ Universitat de Valencia, Valencia, España \\ valeraluism@gmail.com \\ Martínez Usarralde, María Jesús \\ Universitat de Valencia, Valencia, España \\ m.jesus.martinez@uv.es
}

\section{Resumen}

En el mundo globalizado en el que nos encontramos, la integración y la profundización de interdependencias entre las sociedades y las economías involucradas resulta inevitable. Es por ello que se establece la necesidad de crear diálogos interdisciplinares que permitan conocer de parte y parte, legislaciones y políticas sociales que favorezcan el crecimiento mutuo, retroalimentando sociedades que se encuentran esencialmente hiperconectadas.

De esta forma se plantea como muestra de esta necesaria dialéctica, desarrollar un estudio que permita, a través de una metodología comparada, velar por una de las aristas de nuestro sistema globalizado que mayor atención y protección necesita, la infancia. El modelo tradicional de atención a la infancia y la concepción del menor, han sufrido grandes cambios en nuestro país en los últimos años, existiendo una nueva definición que sitúa a los menores como sujetos activos de derechos y donde las medidas de protección que se articulan en la legislación actual, emergen del interés superior del menor. Es por ello que, partiendo de lineamientos regidos por políticas supranacionales, estableceremos como unidades de análisis los sistemas de protección de menores de dos países que política, social e históricamente han permanecido unidos: Colombia y España, centrando nuestra atención en el marco conceptual y legislativo de ambos países. A través de un diálogo comparativo entre ambos sistemas de protección, reconocemos articulaciones legales con preceptos comunes, cuyas actuaciones comparten una base epistemológica y una finalidad en última estancia, la protección del menor. Sin embargo, existen otras medidas cuya mirada debe establecerse desde un proceso de aprendizaje y construcción, que parten de la diferencia pero cuya finalidad debe ser la creación de procesos más cercanos, más humanos, más inclusivos para los protagonistas de estas acciones: los niños, niñas y adolescentes y sus respectivas familias.

\section{Abstract}

The globalized world in which we find ourselves, the integration and deepening of interdependencies between the societies and economies involved is inevitable. That is why it establishes the need to create interdisciplinary dialogues that allow us to know both parts, laws and social policies that support mutual growth, providing feedback to societies that are essentially hyperconnected.

In this way, it is proposed as a sample of this dialectical need, to develop a study that allows, through a comparative methodology, a global approach to the characteristics of our globalized system that needs more attention and protection, childhood. The traditional model of attention to childhood and the conception of the child have undergone great changes in our country in recent years, there is a new definition that places minors as active subjects of rights and where the protection measures articulated in current legislation, emerging from the best interests of the child. Thus, starting from guidelines governed by supranational policies, we will establish as units of analysis the protection systems of minors from countries that are in politics, socially and historically, we have permanently united: Colombia and Spain, focusing our attention on the conceptual framework and legislative of both countries.

Through a comparative dialogue between both systems of protection, we recognize legal articulations with common precepts, whose actions share an epistemological basis and a final purpose, the protection of the minor. However, there are other measures whose vision should be established from a learning and construction process, which starts from the difference but whose purpose should be the creation of closer, more human, more inclusive processes for the protagonists of these actions: children and their respective families.

Palabras clave: Infancia, adolescencia, protección de menores, inclusión, metodología comparada.

Keywords: Childhood, adolescence, child protection, inclusion, comparative methodology. 


\section{INTRODUCCIÓN}

Históricamente se ha concebido al menor ${ }^{1}$ desde diferentes perspectivas, definiendo tanto el concepto como las implicaciones que esta condición trae consigo, especialmente el aspecto educativo (bien sea desde una visión formal, o bien desde el ámbito familiar) como proceso por el que necesariamente debieran pasar todos los niños en su proceso de alcanzar la adultez. Las percepciones y consideraciones en torno a la primera infancia han cambiado ampliamente a lo largo del tiempo en las sociedades en función de los diversos momentos históricos, y se ha forjado una representación social concreta de la infancia a partir de los mismos (Ancheta, 2008).

Reflejo de esta variación conceptual basada en constructos sociales, encontramos como en la Grecia antigua, siguiendo al propio Aristóteles, el infante era considerado un pequeño-hombre que necesitaba la ayuda de un adulto para valerse y cuya voz no ameritaba ser escuchada (Enesco, 2001). En la antigua Roma, los niños y niñas dependían de sus padres y en consecuencia debían someterse a su ley. En la Edad Media, la visión hacia la infancia adquiere un sentido de protección gracias a la propuesta del cristianismo de asumir al hombre como hijo de Dios (Castillo-Gallardo, 2016).

Ya en los siglos XVII y XVIII la infancia fue reconocida como una etapa de la vida del ser humano (Noguera, 2003). Así, frente a la perspectiva medieval del niño como un adulto en miniatura, Rousseau sostiene que es un ser con características propias, que sigue un desarrollo físico, intelectual, moral, reflejándose estas ideas en la frase: «El pequeño del hombre no es simplemente un hombre pequeño» (Rousseau, en Enesco, 2001). No es hasta el S. XIX cuando la concepción del niño comienza a distinguirse del adulto, pues hasta ese momento, el niño se encuentra relegado a una visión de propiedad privada por parte de los padres, por lo que el Estado nada tenía que hacer en esa relación de posesión de un bien familiar, como eran los hijos (Cánovas y Sahuquillo, 2014).

Sin embargo, todavía existen concepciones no unificadas sobre el niño, y no es hasta bien entrado el S. XX donde encontramos la unidad de criterios sobre la concepción del niño, pues hasta entonces, la infancia no es plena y explícitamente reconocida como periodo con sus propias características y necesidades, el niño como persona, con derecho a la identidad personal, a la dignidad y la libertad. Este hecho tiene lugar a partir de la Declaración de los Derechos del Niño, del 20 de Noviembre de 1959, sin embargo, no será hasta 1989 cuando tenga lugar la Convención de los Derechos del Niño (CDN), y se produzca el reconocimiento del menor de edad como objeto social de protección, siendo de obligado cumplimiento para todos los Estados miembros de la Asamblea General de la ONU.

Así, la Convención sobre los Derechos del Niño establece en su artículo 4, la obligación de los Estados Parte de adoptar todas las medidas administrativas, legislativas y de otra índole, que tengan por objeto otorgar efectividad a los derechos reconocidos a los niños y niñas en el referido instrumento internacional. Los países signatarios de la referida Convención, entre ellos los que se encuentran los países objeto de estudio de la presente investigación, han armonizado sus legislaciones internas a los estándares de la CDN, comprometiéndose a desarrollar todo un entramada legislativo para la efectiva protección a la infancia (Morlachetti, 2013).

Según UNICEF (2010), la perspectiva actual reconoce a los niños, niñas y adolescentes como ciudadanos, lo cual supone un reconocimiento donde adultos y menores cuentan con los mismos derechos y donde la infancia cuenta con otros derechos especiales por tratarse de personas en crecimiento. Así, «los niños son diferentes, pero no «menos que» los adultos, y desarrollan también estrategias de interacción con los otros, expresan intereses, necesidades y desacuerdos o satisfacciones» (Ramiro y Alemán, 2016, p. 16). En la actualidad, las necesidades vitales de los menores son concebidas como derechos intrínsecos, donde el Estado vela por el ejercicio pleno de los mismos, además de satisfacerlos plenamente, y no como carencias a subsanar o satisfacer.

\footnotetext{
${ }^{1}$ A lo largo del presente trabajo se usará el término menor para hacer alusión de forma jurídica, a toda persona con una edad inferior a los 18 años, siendo considerado sujeto activo titular de derechos (Cardona, 2012).
} 


\section{PLANTEAMIENTO DE LA INVESTIGACIÓN: PROPUESTA METODOLÓGICA COMPARADA Y ANÁLISIS DE LOS PAÍSES OBJETO DE ESTUDIO}

Seguidamente abordaremos el planteamiento de nuestra investigación, que se desarrolla a partir de los planteamientos teóricos expuestos en la primera parte del trabajo y que toma como referencia el método comparado para el análisis exhaustivo de los diferentes elementos revisados.

\subsection{Propuesta metodológica: método comparado}

El método comparado utilizado se basa en una adaptación de la metodología de García Garrido (1990) y Ferrer (2002), que contempla tres fases: 1. Estudio descriptivo («fase analítica»), en el que se describirán los dos sistemas de protección a la infancia de Colombia y España y se acomete un análisis formal y material, además de explicativo, al tiempo que se delimitan y clasifican las categorías de análisis; 2. Estudio comparativo («fase sintética»), en el que los datos son seleccionados y yuxtapuestos en una tabla, a fin de facilitar su comparación, y, finalmente, la «fase de conclusiones comparadas», en la que se reflexionará sobre los resultados hallados, al tiempo que se sugerirán vías por las que seguir analizando e investigando sobre diferentes aspectos de los sistemas de protección a la infancia.

\subsection{Propuesta metodológica: método comparado}

En este primer momento del método, como ya se ha señalado, se ofrece un análisis descriptivo de los dos sistemas de protección a la infancia seleccionados, correspondientes a los países seleccionados para nuestro estudio: España y Colombia. Una vez presentados, se procederá a la selección de los criterios de comparación, los cuales serán presentados para facilitar su comprensión.

\subsubsection{Sistema de protección a la infancia en España}

A pesar de que ciertos autores, como Francisca Fariña (2000), sitúan el inicio de la protección de menores en España a mediados del S.XIX, se tiene constancia de la existencia de instituciones relativas a la protección de la infancia que son anteriores a dicha fecha. Así, en el año 1337, bajo el reinado de Pedro IV El Ceremonioso, se crea en Valencia la figura del «Pare d'orfens» (Padre de huérfanos) y que se va generalizando posteriormente con otras denominaciones al resto del país. Por ejemplo, en Castilla se denominaría Padre General de Menores, que se integraba en la Junta General Central de la Beneficencia del Reino. En otros lugares aparece el Padre de Mozos o Padre de Vagos, que intentaban cuidar que no aparecieran hábitos considerados como holgazanería y vagabundeo en los menores (Pedreira Massa, 1992). El periodo comprendido entre los siglos XV y XVIII viene determinado por la relegación del menor al ámbito privado o religioso, y la aparición de los hospicios (para menores no delincuentes) y las cárceles (para menores delincuentes).

Sin embargo, no fue hasta el S. XIX cuando surgieron las primeras leyes relacionadas con la protección de menores. Dentro del marco histórico de la Revolución industrial, se crea la Ley de 24 de julio de 1873 o la Ley de 28 de julio de 1878, ambas referidas al trabajo infantil, o la conocida Ley Moyano de 1857 referida a la educación de los menores. No es hasta entrado el siglo XX cuando encontramos la primera Ley de Protección a la Infancia (1904), conocida como la Ley Tolosa, la cual se centraba principalmente en la protección a la salud física y moral del niño, y la vigilancia de todo lo que hiciera referencia a la vida infantil hasta los 10 años, adoptando un carácter higienista y sanitario (Santos Sacristán, s.f.). Con ella se creó el Consejo de Protección a la Infancia y Represión de la Mendicidad, organismo constituido en juntas provinciales y locales para velar por la infancia en todo el territorio.

La regulación más actual de la protección a la infancia puede situarse en la Declaración de Ginebra de 1924 sobre los Derechos del Niño y más recientemente, en la Declaración de los Derechos del Niño de 20 de noviembre de 1959. Con ello, el punto de partida de la regulación normativa vigente en materia de protección de menores en España, se encuentra en la constitución Española de 1978, concretamente en el artículo 39, donde se establece la necesidad de protección a la familia por parte de los poderes públicos, y la garantía de protección de los niños prevista en los acuerdos internacionales que velan por sus derechos. 
Estos principios, reconocidos en la Convención de los Derechos del Niño aprobada por la Asamblea General de las Naciones Unidas de 20 de noviembre de 1989 (ratificada por España el 30 de noviembre de 1990), aparecen articulados en nuestra legislación en la Ley Orgánica 1/1996, de 15 de enero, de Protección Jurídica del Menor y en los preceptos de nuestro Código Civil, donde se establece el concepto de minoría de edad (artículo 315 del Código Civil), y el concepto de menor, como sujeto de protección y donde prime el interés superior del mismo. De igual modo, este sistema de protección es gestionado por las Comunidades Autónomas. Ello queda dispuesto en la Ley Orgánica 1/1996, de 15 de enero, de Protección Jurídica del Menor, de modificación parcial del Código Civil y de la Ley de Enjuiciamiento Civil: «cuando se hace referencia a competencias [...] se especifica que las mismas corresponden a las Comunidades Autónomas y a las ciudades de Ceuta y Melilla, de conformidad con el reparto constitucional de competencias y las asumidas por aquéllas en sus respectivos Estatutos». A nivel nacional se crea en el año 2011 la institución del Ministerio de Sanidad, Servicios sociales e Igualdad, como entidad de referencia ante las actuaciones de las diferentes autonomías en materia de protección al menor.

En el año 2015 se sancionan dos leyes que modifican la Ley 1/1996. Por un lado encontramos la Ley Orgánica 8/2015, de 22 de julio, de modificación del sistema de protección a la infancia y a la adolescencia, que entre otras medidas destacan las modificaciones terminológicas, sustituyéndose el término «deficiencia» por el de «discapacidad» y el término «juicio» por el de «madurez», entre otras.

Por otro lado, encontramos la Ley 26/2015, de 28 de julio, de modificación del sistema de protección a la infancia y a la adolescencia, con ella se logran adaptar los principios de actuación administrativa a las nuevas necesidades que presenta la infancia y la adolescencia en España, tales como la situación de los menores extranjeros, los que son víctimas de violencia y la regulación de determinados derechos y deberes.

\subsubsection{Sistema de protección a la infancia en Colombia}

Colombia es uno de los países que se acoge a la normatividad internacional y a la política pública social vigente para la protección a la infancia, tanto es así que a la fecha, este país cuenta con un importante marco normativo en esta materia. Pero llegar al momento en el que la legislación colombiana en materia de infancia se encuentra hoy en día, ha costado históricamente un largo proceso de profundos cambios, sobre todo a nivel social y con ello, de concepción de la infancia.

Colombia, y en general América Latina, constituye uno de esos casos en que los niños y las niñas hacen parte de la fuerza productiva familiar y sus condiciones de infantes se ven afectados al asumir roles y responsabilidades de adultos. En este sentido, el tratamiento de la infancia en Colombia ha sufrido una serie de cambios históricos y culturales, desde la llegada e imposición de la sociedad europea con el «Descubrimiento», la introducción de elementos como la esclavitud, debiendo las comunidades del Nuevo Mundo modificar sus hábitos y costumbres, para darle paso a las nuevas formas de relacionarse con el mundo establecidas por Occidente (Palacios-Serres, 2004).

Así, entre los siglos XV y XIX, las problemáticas de la infancia unidas a los cambios históricos, políticos y sociales, hicieron que niños y niñas se viesen involucrados en situaciones que necesitaron de una protección especial pasados los años, tales como el trabajo infantil, abuso y explotación sexual, niños y niñas guerrilleros, tráfico de menores, menores militarizados. A mediados del siglo XX, se crea el primer Consejo Nacional de Defensa y Rehabilitación del Menor (Ley 83 de 1946), donde la preocupación por la infancia abandonada y desprotegida comienza a cobrar importancia, creándose además la figura del Juez de Menores. Posteriormente a este consejo, se instaura el Consejo Colombiano de Protección Social del Menor y de la Familia (Decreto 1818 DE 1964), cuya finalidad será la de asegurar dentro del territorio nacional el bienestar y la protección social tanto de la familia como del menor. Para el cumplimiento de esta disposición, el gobierno colombiano crea el Instituto Colombiano de Bienestar Familiar (ICBF) mediante la Ley 75 de 1968, y cuya vigencia continúa hasta la actualidad. El Instituto Colombiano de Bienestar Familiar es el órgano articulador y rector del Sistema Nacional de Bienestar Familiar, y tiene a su cargo la articulación de las entidades responsables de la garantía de los derechos, la prevención de su vulneración, la protección y el restablecimiento de los mismos, en los ámbitos nacional, departamental, distrital, municipal y resguardos o territorios indígenas. (Art 205). En la actualidad, el Instituto Colombiano de Bienestar Familiar está presente en todas las capitales de departamento con sedes regionales, atiende a las poblaciones vulnerables en todo el país con 200 centros zonales. 
La Constitución Política de Colombia de 1991, que declara al país como un estado social de derecho, en sus artículos artículo $44^{\circ}, 67^{\circ}$, hace referencia directa a la protección de los niños y niñas, lo que implica una ratificación de la Convención de1989 y establece un orden jurídico específico para los niños y niñas.

Finalmente, y como resultado de las diferentes medidas adoptadas y moldeadas por Colombia para la protección a la infancia, se dicta el Código de Infancia y Adolescencia, mediante Ley 1098 de 2006, cuya finalidad será la de "garantizar a los niños, a las niñas y a los adolescentes su pleno y armonioso desarrollo para que crezcan en el seno de la familia y de la comunidad, en un ambiente de felicidad, amor y comprensión» (Art. 1, Código de Infancia y Adolescencia).

A partir de esta descripción, la categoría seleccionada será la siguiente:

- Historia, legislación, instituciones de la protección a la infancia y conceptualización del menor de edad sujeto de derechos y objeto de protección.

De esta categoría, a su vez, se deducen diferentes apartados organizados en indicadores, para una mayor aproximación a la realidad que abordamos desde el presente estudio.

\subsection{Fase sintética: yuxtaposición de los datos ofrecidos}

A continuación se yuxtaponen los datos ofrecidos desde los dos sistemas de protección a la infancia, a fin de detectar cuáles son los aspectos coincidentes y las señas idiosincrásicas de cada uno de ellos, a partir de la fase anterior.

\subsubsection{Historia, legislación, instituciones de la protección a la infancia y conceptualización del menor de edad sujeto de derechos y objeto de protección}

La tabla que se presenta a continuación recoge los aspectos fundamentales que sirven de «carta de presentación» a esta figura: orígenes, legislación que avala los sistemas de protección a la infancia de ambos países que se van a comparar, distribución de competencias en cada uno de los sistemas determinadas por la legislación presentada, instituciones competentes en cada caso y finalmente los conceptos que presentan cada uno de los sistemas de protección, para referirse al menor de edad sujeto de derechos y objeto de protección. 
Tabla 1. Yuxtaposición de historia, legislación, instituciones de la protección a la infancia y conceptualización del menor de edad sujeto de derechos y objeto de protección de los sistemas de protección de menores de España y Colombia.

\begin{tabular}{|c|c|c|}
\hline & $\begin{array}{l}\text { SISTEMA DE PROTECCIÓN DE } \\
\text { MENORES DE ESPAÑA }\end{array}$ & $\begin{array}{l}\text { SISTEMA DE PROTECCIÓN DE } \\
\text { MENORES DE COLOMBIA }\end{array}$ \\
\hline $\begin{array}{l}\text { Hitos que fundamentan } \\
\text { los sistemas de } \\
\text { protección }\end{array}$ & $\begin{array}{c}\text { Trabajo infantil. } \\
\text { Infancia abandonada. } \\
\text { Maltrato infantil. } \\
\text { Mendicidad. }\end{array}$ & $\begin{array}{c}\text { Colonización. } \\
\text { Trabajo infantil. } \\
\text { Abuso y explotación sexual. } \\
\text { Niños y niñas guerrilleros. } \\
\text { Tráfico de menores. } \\
\text { Menores militarizados }\end{array}$ \\
\hline $\begin{array}{l}\text { Primeras medidas de } \\
\text { protección a la infancia. }\end{array}$ & $\begin{array}{c}\text { Año 1337: creación de la figura del «Pare } \\
\text { d'orfens» (Padre de huérfanos). }\end{array}$ & $\begin{array}{l}\text { Año 1946: creación del Consejo Nacional de } \\
\text { Defensa y Rehabilitación del Menor. }\end{array}$ \\
\hline $\begin{array}{l}\text { Legislación } \\
\text { vigente }\end{array}$ & $\begin{array}{c}\text { Ley Orgánica } 1 / 1996 \text { de Protección } \\
\text { Jurídica del Menor. } \\
\text { Modificada mediante Ley Orgánica } 8 / 2015 \text {, } \\
\text { de } 22 \text { de julio, de modificación del sistema } \\
\text { de protección a la infancia y a la } \\
\text { adolescencia, y Ley } 26 / 2015 \text {, de } 28 \text { de } \\
\text { julio, de modificación del sistema de } \\
\text { protección a la infancia y a la adolescencia. }\end{array}$ & $\begin{array}{l}\text { Ley } 1098 \text { de } 2006 \text {, Código de } \\
\text { Infancia y Adolescencia. } \\
\text { Modificada mediante Ley } 1878 \text { del } 9 \text { de } \\
\text { Enero de } 2018 .\end{array}$ \\
\hline Institución responsable & $\begin{array}{c}\text { Ministerio de Sanidad, Servicios Sociales } \\
\text { e igualdad. Delega en las Comunidades } \\
\text { Autónomas. }\end{array}$ & Instituto Colombiano de Bienestar Familiar \\
\hline Competencia territorial & Autonómica & Nacional \\
\hline $\begin{array}{l}\text { Concepto explícito en la } \\
\text { legislación. }\end{array}$ & $\begin{array}{l}\text { Infancia y Adolescencia. } \\
\text { Menor. }\end{array}$ & $\begin{array}{c}\text { Infancia y Adolescencia. } \\
\text { Niños, Niñas y Adolescentes. }\end{array}$ \\
\hline
\end{tabular}

Fuente: elaboración propia.

Como podemos observar, los dos sistemas de protección a la infancia, toman como punto de partida diferentes problemáticas que afectan directamente a la vida e integridad de la infancia, pero observamos como las fechas en las que aparecen las primeras medidas de protección son muy distantes en el tiempo, surgiendo en España en el siglo XIV y en Colombia en el siglo XX.

En cuanto al ámbito legislativo, debemos señalar que si bien las fechas de las leyes vigentes en materia de protección a la infancia son distintas, puede apreciarse como en los últimos años existe una tendencia que marca la modificación e introducción de nuevos preceptos en las leyes anteriores, articulándose así medidas que contemplen las necesidades actuales de la infancia. 
En relación a la competencia territorial, apreciamos como en España impera un modelo descentralizado, donde las competencias son distribuidas por el Estado, mediante el Ministerio de Sanidad, Servicios Sociales e Igualdad, a las diferentes autonomías. Sin embargo, en Colombia, contamos con una única institución a nivel nacional, el Instituto Colombiano de Bienestar Familiar, que está presente en cada una de las principales ciudades y capitales de departamento del país, manejando un modelo único y centralizado de competencias, distribuido por todo el territorio nacional.

Finalmente, en relación a los conceptos manejados por ambas legislaciones, en España se aprecia la tendencia al uso del término jurídico «menor» para hacer alusión a niños, niñas y adolescentes menores de 18 años, destinatarios de la ley vigente. En Colombia encontramos un uso generalizado de los términos «niño, niña y adolescente» para referirse a los destinatarios de la ley, manejando un lenguaje más inclusivo, que reconoce y hace visibles a las diferentes etapas de edad implicadas como el reconocimiento de ambos géneros.

\section{DISCUSIÓN Y CONCLUSIONES}

Como conclusión a los sistemas de protección de menores escrutados, así como su interpretación, hemos podido comparar los hallazgos encontrados a fin de dilucidar sus puntos de convergencia, y de comprender la estructura y organización en torno a las legislaciones vigentes en materia de infancia. Desde este marco, la intención del presente trabajo se ha identificado con hallar los aspectos más significativos de cada uno de dichos sistemas y analizar cómo las mismas finalidades pueden estructurarse de forma diferenciada, llegando incluso a apreciar cómo una terminología puede considerarse más apropiada que otra, a la luz de hacer más inclusivos si cabe los sistemas de protección a la infancia.

Uno de los aprendizajes más importantes que puede extraerse a partir de nuestro estudio comparado, es que podemos contemplar cómo los orígenes de ambos sistemas de protección tienen un punto en común, pero surgen de problemáticas diferenciadas, en fechas alejadas en el tiempo, y cómo uno puedo influir en el otro, a partir de la época de la colonización y el cambio de visión radical de la infancia en la etapa pre-colonial.

Ante este panorama de entramados legales, sociales y culturales que afectan a la infancia de ambos países y a la construcción de los sistemas de protección de menores, el presente estudio evidencia una pequeña parte del tejido común que une a ambos países, sin embargo, debe darse continuidad al mismo, para lograr dar voz a los diferentes agentes implicados en los procesos que nos ocupan, así como profundizar en el conocimiento de políticas sociales, medidas, profesionales, mecanismos, entre otros elementos, que dotan de sentido a los sistemas de protección a la infancia.

\section{REFERENCIAS BIBLIOGRÁFICAS}

Ancheta, A. (2008). Hacia una nueva concepción de la educación de la primera infancia como derecho: avances y desafíos globales. Revista Iberoamericana de Educación, vol. 47(5), 1-12.

Cánovas, P. y Sahuquillo, P. (2014). Familias y menores: retos y propuestas pedagógicas. Valencia: Tirant Humanidades.

Cardona, J. (2014). El interés superior del niño: balance y perspectivas del concepto en el $25^{\circ}$ aniversario de la Convención sobre los Derechos del Niño. Revista española de desarrollo y cooperación. Vol. 34, p. 21-40.

Castillo-Gallardo, P. (2016): Historia de la Infancia observada desde los ejes del Juego, Juguete y Desigualdad, Educação em Foco, 20(3), pp. 289-322.

Constitución española (1978). Congreso de los Diputados, Madrid, España, 6 de Diciembre de 1978. 
Constitución política colombiana (1991). Asamblea Nacional Constituyente, Bogotá, Colombia, 6 de Julio de 1991.

Decreto 1818 de 1964, 17 de julio. Por el cual se crea el Consejo Colombiano de Protección Social del Menor y de la Familia, se reorganiza la actual División de Menores del Ministerio de Justicia y se dictan otras disposiciones. Diario oficial No. 34.497 de 26 de octubre de 1964.

Enesco, I. (2001). El concepto de infancia a lo largo de la historia. Ensayo, Madrid. Universidad Complutense de Madrid.

Fariña F. y Arce R. (coords.), (2000). Psicología jurídica al servicio del menor. Barcelona: Cedecs. Ferrer, F. (2002): La educación comparada actual. Madrid: Ariel.

García, J.L. (1990): Fundamentos de la educación comparada. Madrid: Dykinson.

Ley 1098 de 2006, 8 de noviembre. Por la cual se expide el Código de la Infancia y la Adolescencia. Diario Oficial No. 46446 del 08 de noviembre de 2006. Congreso de Colombia.

Ley 1878 de 2018, de 8 de enero, por medio de la cual se modifican algunos artículos de la ley 1098 de 2006, por la cual se expide el código de la infancia y la adolescencia, y se dictan otras disposiciones. Congreso de Colombia.

Ley 26/2015, de 28 de julio, de modificación del sistema de protección a la infancia y a la adolescencia. Boletín Oficial del Estado. Número 180, de 29 de Julio de 2015, pp. 64544 a 64613.

Ley 75 de 1968, de 30 de diciembre, por la cual se dictan normas sobre filiación y se crea el Instituto Colombiano de Bienestar Familiar. Diario Oficial No. 32.682 de 31 de diciembre de 1968. Congreso de Colombia.

Ley 83 de 1946, de 26 de diciembre. Diario Oficial No. 26.363, de 24 de febrero de 1947. Congreso de Colombia.

Ley Orgánica 1/1996, de 15 de enero, de Protección Jurídica del Menor, de modificación parcial del Código Civil y de la Ley de Enjuiciamiento Civil. Boletín Oficial del Estado. Número 15, de 17 de Enero de 1996.

Ley Orgánica 8/2015, de 22 de julio, de modificación del sistema de protección a la infancia y a la adolescencia. Boletín Oficial del Estado. Número 175, de 23 de Julio de 2015.

Morlachetti, A. (2013): Sistemas nacionales de protección integral de la infancia. Fundamentos jurídicos y estado de aplicación en América Latina y el Caribe. Santiago de Chile: CEPAL. Recuperado de: https://repositorio.cepal.org/ handle/11362/4040.

Noguera, C. (2003): Reflexiones sobre la desaparición de la infancia. Pedagogía y Saberes, 18, pp. 75-82.

Palacios-Serres, C. (2004). El descubrimiento de América, pasado y porvenir de la infancia de este continente. Recuperado de: http://www.carmenpalaciosserres.com/docs/infancia/historia.pdf.

Pedreira-Massa, J.L. (1992). Historia de la legislación para la infancia en España: Una revisión crítica. Revista de la Asociación Española de Neuropsiquiatría. Vol. XII, No. 42, pp. 215-220.

Ramiro, J. y Alemán, C. (2016). ¿El surgimiento de un nuevo sujeto de ciudadanía? Aportaciones teóricas al debate contemporáneo sobre los derechos de los niños. Papers: Revista de sociologia, Vol. 101 Núm. 2, p. 169-193.

Santos Sacristán, M., (s.f.). Los inicios de la protección a la infancia en España 1873-1918. Recuperado de: www.um.es/ ixcongresoaehe/pdfB3/Los\%20inicios\%20de\%20la\%20proteccion\%20infancia.pdf.

UNICEF (2008). Estado mundial de la infancia. Conmemoración de los 20 años de la Convención sobre los Derechos del Niño. Nueva York: UNICEF. 